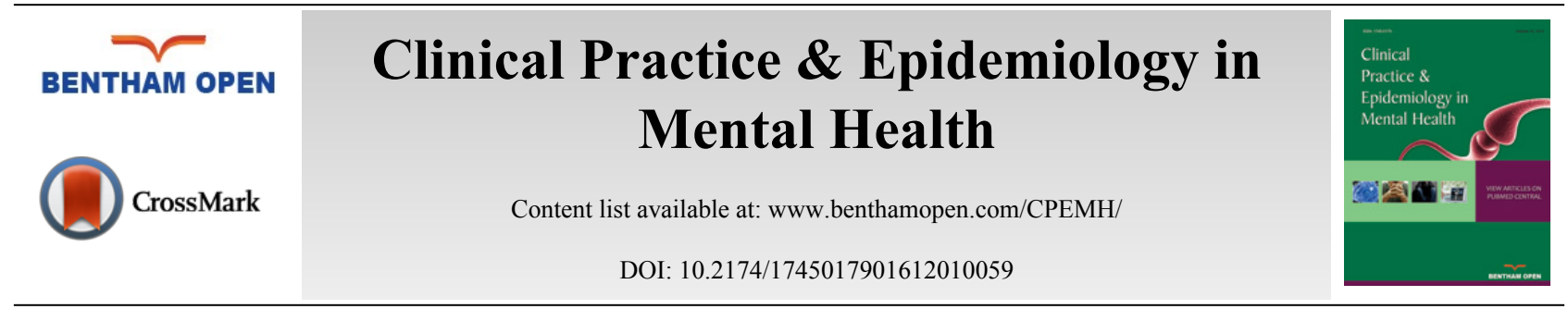

RESEARCH ARTICLE

\title{
How Stable are Temperaments in the Clinical Setting: A Pilot Study
}

Elie G. Karam ${ }^{\mathrm{a}, \mathrm{b}, *}$, Elaine E1 Khoury ${ }^{\mathrm{a}}$ and Lynn Itani ${ }^{\mathrm{a}}$

${ }^{a}$ Institute for Development, Research, Advocacy \& Applied Care (IDRAAC), Beirut, Lebanon

${ }^{b}$ Department of Psychiatry \& Clinical Psychology, St. George Hospital University Medical Center, Balamand University, Faculty of Medicine, Beirut, Lebanon

Received: June 01, 2016

Revised: July 21, 2016

Accepted: August 02, 2016

\section{Abstract:}

\section{Background:}

An essential point in evaluating the utility of measuring temperaments is the stability of the instrument used especially in the presence of mental disorders. One of the most commonly used instruments in the clinical setting is the Temperament Evaluation of Memphis, Pisa, Paris and San Diego Auto-questionnaire (TEMPS-A). To our knowledge, the TEMPS-A's stability in an outpatient adult clinical setting has not been evaluated.

\section{Objective:}

To assess the stability of the effect of temperament, time and clinical intervention.

\section{Methods:}

A sample of 89 adult outpatients was assessed at baseline and follow-up on their TEMPS-A scores. Diagnoses of mental disorders were reached through clinical interviews, and the severity of the conditions was clinically assessed at baseline and follow-up on a Likert scale. Changes in scores were examined in terms of z-scores, and possible predictors of the change in scores were assessed.

\section{Results:}

Eighty-nine percent of all subjects' temperaments scores did not change or changed less than one z-score, and specifically: $84.2 \%$ in the case of depressive, $89.9 \%$ for cyclothymic, $92.1 \%$ for hyperthymic, $92.2 \%$ for irritable, and $86.5 \%$ for anxious temperaments. For all of the five temperaments, age, gender, time difference between baseline and follow up, number of diagnoses, and percent improvement were not significantly associated with the change in temperament scores.

\section{Limitations:}

Well-established severity measures would add to the validity of any future findings.

\section{Conclusion:}

Shifts in temperament scores between baseline and follow-up were minor, thus proving the stability of temperaments and the TEMPS-A scale in a clinical setting.

Keywords: Anxious, Cyclothymic, Dysthymic, Hyperthymic, Irritable, Temps A.

\section{HIGHLIGHTS}

- Temperament stability in an outpatient adult clinical setting has not been studied.

\footnotetext{
* Address correspondence to this author at the IDRAAC (Institute for Development Research, Advocacy, \& Applied Care), Department of Psychiatry and Clinical Psychology, St. George Hospital University Medical Center, Faculty of Medicine, Balamand University, P.O. Box: 166227 Ashrafieh, Beirut 1100 2110, Lebanon; Tel: + (961) 1 583583; Fax: + (961) 1 587190; E-mail: egkaram@idraac.org
} 
- $89 \%$ of subjects' TEMPS-A scores did not change or changed less than one z-score.

- None of factors studied were associated with the change in temperament scores.

\section{INTRODUCTION}

Temperament has been an area of interest for many researchers trying to link the relation of "constitutional cores" to consequential mental health disorders. Instruments have been developed trying to find a universal measure of temperament; one of the most commonly used in clinical setting being the Temperament Evaluation of Memphis, Pisa, Paris and San Diego Auto-questionnaire (TEMPS-A) [1]. It has been used in epidemiological and clinical studies as a self-filled questionnaire for adults (age $\geq 18$ ) in many countries and has been translated into several languages from its original English language [2] including Lebanese Arabic [3]. TEMPS-A measures individuals on five temperaments: dysthymic (DT), cyclothymic (CT), hyperthymic (HT), irritable (IT), [4] and anxious (AT), which was the last added to the original four temperaments.

An essential point in evaluating the utility of measuring temperaments is the stability of the instruments used to measure it especially in the presence of other disorders, such as mental disorders. In an attempt to explore the characteristics of the Lebanese temperament profile, but also to assess the stability of the instrument, the LebaneseArabic TEMPS-A was administered in an epidemiological setting as part of the Lebanese Evaluation of the Burden of Ailments and Needs Of the Nation (L.E.B.A.N.O.N), a national study conducted all over Lebanon.

Ninety percent of subjects in this national study stated that their responses on the TEMPS-A characterized them most of the time since the age of $18 \mathrm{y}$ [5]. The stability of the TEMPS-A in adults was, to our knowledge, directly evaluated only once previously. This was a prospective study on a non clinical population of white collar workers in Japan $(n=178)$ who were free from present or past psychiatric diagnosis and the sample was tested twice at six year intervals. The study's conclusion stated that the temperaments exhibited good long-term stability and were robust, irrespective of temperament type, gender and age [6]. This brief report, explored prospectively the TEMPS's stability in an outpatient adult clinical setting. This paper reports the stability of each temperament (in its subscales) as measured prospectively and examines the roles of age, gender, time between baseline and follow-up, as well as clinical diagnosis and most importantly, the role of improvement of disorder(s) for which the patients were being treated with changes, if any, in the temperament profiles.

\section{METHODS}

\section{Instruments}

Consecutive patients were recruited from the outpatient unit of the Medical Institute for Neuropsychological Disorders (MIND), at the Psychiatry department of the St. Georges Hospital University Medical Center (SGHUMC)/, a sister institution of the Institute for Development, Research, Advocacy and Applied Care (IDRAAC). A sample of 89 consecutive outpatients aged above 18 was assessed at baseline and again in the follow-up on their 1) temperaments scores using TEMPS-A in Lebanese Arabic [3], English [7], or French [1] depending on the patients' preference and 2) clinical rating of the severity of their mental health condition using a five point Likert scale with the following categories: very bad, bad, medium, good, and very good (one being the least severe).

Diagnoses were reached through interviews, first by a physician assistant using DSMIV criteria and then upon reinterview by the psychiatrist (EGK). This is the conventional way the outpatient intake and follow-up of patients are done in our department.

\section{Variables}

\section{Temperaments}

Scores on the five temperament subscales were used for analysis using TEMPS-A: 21 items on each of DT, CT, HT and IT (20 for males), and 26 items for AT. For analysis, sub-scores were used in two forms; continuous and categorical. The latter was done using total population parameter measures (mean and standard deviations): 7.6 ( \pm 2.9 ) for DT, $5.9( \pm 4.3)$ for CT, $12.5( \pm 4.5)$ for HT, $2.8( \pm 3.1)$ for IT and $6.6( \pm 5.2)$ for AT [3].

\section{Improvement}

Improvement in mental disorders was estimated and used in two ways, 1) continuous form; in percent improvement 
as assessed by comparing their clinical severity assessment scores ( 1 to 5) at baseline and then in the follow-up and in a 2 ) categorical formincluding none ( $<1$ point improvement in scores), moderate ( 1 or 2 points improvement), or a lot ( 3 or more points improvement).

\section{STATISTICS}

Descriptive statistics were used to report on frequencies of categorical variables and means with their standard deviations (SD) for continuous ones. For each of the five sub temperament scales, pairwise t-test was used to test for possible significant change between baseline and follow up scores among participants. Linear regression models were used to test possible predictors of the change in the temperament scores. Analysis was performed using SPSS version 17.0 and $95 \%$ confidence interval was set for significance.

\section{RESULTS}

\section{Sample Characteristics}

The sample's age ranged between 19 and 60 y with a mean of 35.8 years $( \pm 10.6 \mathrm{SD})$, with $50.6 \%$ being males. $31.5 \%$ had one mental disorder, $39.3 \%$ had two, $21.3 \%$ had three, $6.7 \%$ had four and $1.1 \%$ had five mental disorders. The two most common disorders were major depression (48\%) followed by anxiety disorders $(40 \%)$. The rate of other disorders was as follows: bipolar disorder (20\%), OCD (16\%), all psychotic disorders (14\%), ADD (5\%), substance use disorder $(5 \%)$, and other $(4 \%) .54 \%$ of the sample had a follow-up period of six months or more, $14 \%$ had 3 to 6 months, $10 \%$ had $2-3$ months, and $22 \%$ had less than 2 months.

\section{Improvement of Disorders at Follow-up}

A total of 70 patients had complete data on the severity of their mental health condition scored globally as very bad, $\mathrm{bad}$, or medium at baseline by the clinicians. Out of those, 44.3\% (31/70) improved a lot, 41.4\% (29/70) improved moderately and 14.3\% (10/70) did not improve. More patients in the "improved a lot" category had only one diagnosis $(41.9 \%)$, whereas only $10 \%$ of those who improved moderately or "did not improve at all" had only one diagnosis.

\section{Shift in Temperament Scores}

All temperament scores (except the hyperthymic temperament) significantly decreased at follow-up. The mean changes were, however, quite modest 0.9 to 1.6 on total scores for each temperament. These mean changes, as a percentage of total scores of subscales, ranged from 3.3\% (for hyperthymic) to $6.7 \%$ (for cyclothymic). (Table 1) These changes were also examined in terms of z-scores for each temperament. Shifts in temperament scores in z scores were minor too. About half of the sample did not change at all; $89 \%$ of all subjects' temperaments scores did not change or changed less than one z-score $( \pm)$, and specifically $84.2 \%$ in the case of DT, $89.9 \%$ for CT, $92.1 \%$ for HT, $92.2 \%$ for IT, and $86.5 \%$ for AT (Fig. 1).

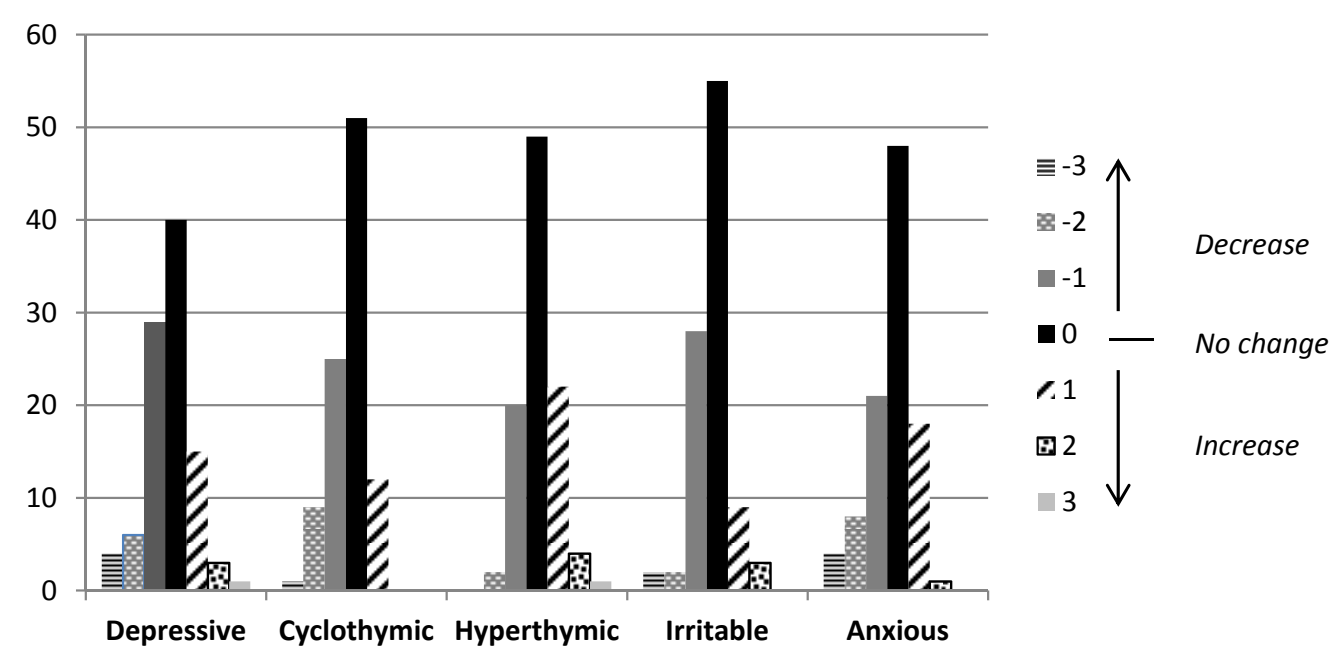

Fig. (1). Percent shift in temperament scores using z-scores between baseline and follow-up. 
Table 1. Temperament mean scores at baseline and follow up $(n=89)$.

\begin{tabular}{|c|c|c|c|c|c|}
\hline \multirow{2}{*}{ Temperament } & \multicolumn{3}{|c|}{ Mean \pm SD } & $\begin{array}{c}\text { P-value for pairwise } \\
\text { comparison }\end{array}$ \\
\cline { 2 - 7 } & Baseline score & Follow up score & Absolute difference in score & Difference as \% of score & \\
\hline Dysthymic & $10.8 \pm 3.4$ & $9.9 \pm 3.4$ & $0.9 \pm 3.4$ & $4.3 \pm 16.2$ & $0.013^{*}$ \\
\hline Cyclothymic & $11.2 \pm 5.5$ & $9.7 \pm 5.5$ & $1.4 \pm 4.1$ & $3.3 \pm 17.6$ & $0.001 *$ \\
\hline Hyperthymic & $9.9 \pm 4.1$ & $10.6 \pm 4.4$ & $0.7 \pm 3.7$ & $5.9 \pm 17.1$ & 0.068 \\
\hline Irritable & $7.0 \pm 4.9$ & $5.8 \pm 4.4$ & $1.2 \pm 3.5$ & $6.2 \pm 21.5$ & $0.001^{*}$ \\
\hline Anxious & $13.3 \pm 6.2$ & $11.7 \pm 6.3$ & $1.6 \pm 5.6$ & & $0.008^{*}$ \\
\hline
\end{tabular}

*significance $(\mathrm{p}<0.05)$

\section{Possible Factors Affecting Change in Temperament Between Baseline and Follow Up}

Linear regression models were used for the change in the temperament scores with the following possible factors: age, gender, time difference between baseline and follow-up, number of diagnoses, and percent improvement controlling for interaction between percent improvement and number of diagnoses. For all of the five temperaments, none of the mentioned variables were significantly associated with change in scores at the bivariate and multivariate analysis.

\section{DISCUSSION}

Assessing temperament is of major help in clinical work as it sheds light on the "usual" affective reactions of individuals. However, since temperaments reflect, to some degree, clinical diagnoses as we now understand them, the possibility remains that temperaments measured while in a state of depression, for example, might change when the depression lifts off. Therefore, in spite of the fact that temperaments were to be observed largely stable in a national study and in another non-clinical study in Japan [6], it was concluded that it is important to explore this stability in a clinical sample. It is to be remembered here that patients filling the TEMPS-A are requested repeatedly to answer the individual temperament items by reflecting on their lifetime (since age 18, and into adulthood) and not only at the time of the interview, thus to ensure that the TEMPS-A reflects traits and not only their present state. This pilot study shows that the mean change in temperament scores is less than $7 \%$ of raw scores for all the temperament sub-scales, albeit with wide variations. Examining these changes in terms of z-scores: about $90 \%$ of patients maintained temperament scores within one z-score (with 50\% not changing at all). There are obvious limitations to this brief report: first, a larger sample might detect changes and assess the scope of variations, in subgroups not obvious in smaller ones. A second limitation is that regular clinical methods were used to establish diagnosis examine and severity of disorders; wellestablished structured interviews and severity measures would add to the validity of any future findings. Notwithstanding these limitations, these findings are encouraging and if confirmed by others, will add to our understanding of traits, such as basic affective temperaments in the final expression of behavior in general and in mental disorders in particular.

\section{CONFLICT OF INTEREST}

The authors confirm that this article content has no conflict of interest.

\section{ACKNOWLEDGEMENTS}

Declared none.

\section{REFERENCES}

[1] Akiskal HS, Akiskal K, Allilaire JF, et al. Validating affective temperaments in their subaffective and socially positive attributes: psychometric, clinical and familial data from a French national study. J Affect Disord 2005; 85(1-2): 29-36. a [PMID: 15780673]

[2] Rihmer Z, Akiskal K, Rihmer A, Akiskal H. Current research on affective temperaments. Curr Opin Psychiatry 2010; 23: 12-8.

[3] Karam EG, Mneimneh Z, Salamoun M, Akiskal KK, Akiskal HS. Psychometric properties of the Lebanese-Arabic TEMPS-A: a national epidemiologic study. J Affect Disord 2005; 87(2-3): 169-83.

[PMID: 16023731]

[4] Akiskal HS, Mallya G. Criteria for the "soft" bipolar spectrum: treatment implications. Psychopharmacol Bull 1987; 23(1): 68-73. [PMID: 3602332] 
[5] Karam EG, Mneimneh ZN, Salamoun MM, Akiskal HS, Akiskal KK. Suitability of the TEMPS-A for population-based studies: ease of administration and stability of affective temperaments in its Lebanese version. J Affect Disord 2007; 98(1-2): 45-53. [PMID: 16949160]

[6] Kawamura Y, Akiyama T, Shimada T, et al. Six-year stability of affective temperaments as measured by TEMPS-A. Psychopathology 2010; 43(4): 240-7. [PMID: 20424505]

[7] Akiskal HS, Akiskal KK, Haykal RF, Manning JS, Connor PD. TEMPS-A: progress towards validation of a self-rated clinical version of the Temperament Evaluation of the Memphis, Pisa, Paris, and San Diego Autoquestionnaire. J Affect Disord 2005; 85(1-2): 3-16. [PMID: 15780671]

(C) Karam et al.; Licensee Bentham Open

This is an open access articles licensed under the terms of the Creative Commons Attribution-Non-Commercial 4.0 International Public License (CC BY-NC 4.0) (https://creativecommons.org/licenses/by-nc/4.0/legalcode), which permits unrestricted, non-commercial use, distribution and reproduction in any medium, provided that the work is properly cited. 